\title{
The initial trauma associated hemorrhagic shock in the multiply injured patient has pro-inflammatory effects. A retrospective single center cohort study
}

\author{
Sandro Michael Heining ${ }^{1}$, Catharina Keller ${ }^{2}$, Hans-Peter Simmen $^{1}$, Hans-Christoph Pape ${ }^{1}$, Kai OliverJensen ${ }^{1}$, Carina Eva Maria Pothmann ${ }^{1}$, \\ Martin Berli $^{3}$, Kai Sprengel ${ }^{1}$ and Ladislav Mica ${ }^{*}$ \\ ${ }^{1}$ Trauma Surgery, University Hospital Zurich, 8091 Zurich, Switzerland \\ ${ }^{2}$ Neurology, Kanstonsspital Winterthur, 8401 Winterthur, Switzerland \\ ${ }^{3}$ Orthopedics, University Hospital Balgrist, 8008 Zurich, Switzerland
}

\begin{abstract}
Background: The development of a Systemic Inflammatory Response Syndrome (SIRS) has been discussed to be related to the trauma load. Trauma, especially the multiple injured patient suffers blood loss in a various extent. The outcome of such patients is also linked with the need for transfusions and SIRS. Therefore, the association between the initial blood loss and the incidence of IL 6 release following multiple injuries was examined.

Methods: Totally 545 patients $\geq 16$ years and with an ISS $>16$ were included into this retrospective cohort study. The sample was divided according to the ATLS shock classes I-IV on admission in the trauma bay and the shock-index $(<0,5 ; 0.5-1.5$ and $>1.5)$ at trauma site. The systemic levels of IL6 were measured 24 and 48 hours after admission. Data were analyzed using Kruskal-Wallis H test. The predictive quality was tested using receiver-operating curves (AUC), the independent predictive power was analyzed using binary logistic regression. The analysis was conducted using IBM SPSS ${ }^{\oplus} 23.0$. Level of evidence III.

Results: IL6 significantly increased within the first $24 \mathrm{~h}$ according to shock (252ng $-872 \mathrm{ng}, \mathrm{p}<0.001)$. There were highly predictive values between IL6 and SIRS (24h) (AUC: $0.345-0.951$ for ATLS shock I-IV, p < 0.001; AUC: $0.443-0.963$ for shock-index $0.5-1.5$, p < 0.001 ). ATLS shock revealed as an independent predictor for IL6 increase after $48 \mathrm{~h}(\mathrm{p}<0.001)$.

Conclusion: Initial shock-index at trauma site and the ATLS shock degree in the trauma bay are high predictors for IL6 release what may serve as a primer for the induction of SIRS. Very early control of blood loss seems to be the key point. However, these results might also point on the change of transfusion protocols in the multiple injured patient.
\end{abstract}

\section{Introduction}

The physiological reaction to severe multiple trauma has been shown to result in the systemic inflammatory response syndrome (SIRS) which appears to be related to trauma severity [1-3]. A sustained systemic inflammation is associated with increased mortality in multiple injured patients and results in the compensatory antiinflammatory response syndrome (CARS) as an initially parallel to SIRS running compensatory part with an immunological burn out and higher susceptibility to infections [3]. There were several trials to determine the origin or the trigger of SIRS during the last decades, though the studies remain descriptive and the initial trigger was not detected [4]. Among the predictors of SIRS, direct indicators such as injury severity (ISS, NISS) and indirect indicators such as the rate of blood transfusions were discussed [5-8]. Certainly, trauma load, blood loss, and amount of transfused blood go hand in hand and are a result of the trauma. IL6 is a pro-inflammatory cytokine secreted by plenty of cells mostly hematopoietic cells including tumor cells maintaining their survival by the JAK/STAT pathway [9]. The IL6 concentration in patient's peripheral blood might mirror the possibility to develop SIRS and multi organ dysfunction in the case of multiple injuries [10]. This retrospective cohort study was designed on the question, whether the initial blood loss at trauma site and then in the trauma bay influences the pro-inflammatory state in the multiple injured patient. The fact is that trauma leads to blood loss and the multiple injured patients bleed in a physiologically relevant way. The question was whether the blood loss classified on the trauma scene by the shock index and in the trauma bay by the ATLS shock degree has a pro-inflammatory effect and an effect on the expression of IL6 in multiple injured patient arising a therapeutic consequence. Here the statistical proof is provided that IL6 is raised by the blood loss in the multiple injured patient.

\section{Methods}

\section{Patient sample}

In this retrospective cohort study, 545 patients with multiple trauma, admitted to the trauma bay of the University Hospital Zurich (Switzerland), were included. Totally, 2995 datasets had to be excluded due to missing IL6 data. The data were included 01.01.2000 - 12.31.2014.

${ }^{\star}$ Correspondence to: Ladislav Mica, PhD, FEBS, Division of Trauma Surgery, University Hospital Zurich, Rämistrasse 1008091 Zurich, Switzerland, Tel: 0041 4425511 11; E-mail: ladmic@gmail.com

Key words: Multiple Injuries, Polytrauma, Hemorrhagic Shock, Interleukin 6, Systemic Inflammatory Response Syndrome, Sepsis, Systemic Inflammation

Received: July 19, 2018; Accepted: July 26, 2018; Published: July 31, 2018 

study

In this period, only 545 multiple injured patients with IL6 data were available. The inclusion criteria were an injury severity score (ISS) $>16$ points, age $\geq 16$ years, and immediate admission to the trauma bay of the University Hospital Zurich after incurring the multiple trauma. The patient sample was divided into four groups according to the ATLS shock classes I-IV and the shock index $<0.5,0.5-1.5$ and $>1.5$ [11].

\section{Data collection}

All data were collected retrospectively according to Good Clinical Practice Guidelines and were pooled in the Zurich Trauma Registry data bank. The patients' data were retrieved from patient records over an time period of 21 days, with the approval of the local institutional review board (IRB), according to the University of Zurich IRB guidelines and the World Medical Association Declaration of Helsinki. The study was conducted according to our institutional guidelines for good clinical practice (Ethics Committee of the University Hospital Zurich Permission: "Retrospektive Analysen in der Chirurgischen Intensivmedizin” Nr. St.V. 01-2008). Level of Evidence III.

\section{Scoring systems}

The scoring systems were used to depict a detailed view on the multiple injured patient sample. The overall physiological impairment was evaluated by the acute physiology and chronic health evaluation (APACHE II) score of the patient at admission [12]. The ISS and the new injury severity scale (NISS) were used to define the severity of trauma [13,14]. The abbreviated injury scale (AIS; update 2008 version) was used to describe injuries in specific anatomical regions. The Trauma Score - Injury Severity Score (TRISS) was used to analyze the probability of death in the patients sample at admission [15]. The Simplified Acute Physiology Score (SAPS II) was calculated according to Le Gall at admission [16]. SIRS was defined as previously published and sepsis was defined as two or more SIRS criteria with an infectious focus [17].

\section{Groups}

To determine and to distinguish the Shock states two different staging methods were chosen and calculated retrospectively from patient's records for descriptive purposes.

1. The ATLS classification (Class I, II, III and IV) for severe hemorrhage was evaluated from the earliest available data at admission in the trauma bay.

2. The shock index according to Allgöwer was calculated from the earliest available data at trauma scene and subdivided into three groups [18]: $<0.5,0.5-1.5$ and $>1.5$.

\section{Laboratory parameters}

Blood lactate levels, $\mathrm{pH}$, and hematocrits were measured at intervals using a blood gas analyzer (ABL800 Flex, Radiometer, Thalwil, Switzerland). The prothrombin time was measured using a standardized method [19].

\section{Statistical analysis}

Missing datasets were excluded. Data are presented as mean \pm standard deviation for continuous variables and as percentages for categorical variables. The Two-tailed Kolmogorov-Smirnov test was used for normality testing if data is $p>0.05$ than it was considered to be normally distributed. The data of the groups were compared using a $\chi^{2}$ test for categorical data. The one-way analysis of variance (ANOVA) was taken for normally distributed continuous data. For not normally distributed continuous data, the Kruskal-Wallis $\mathrm{H}$ test was used. Results were considered significant if $\mathrm{p}<0.05$. If the $\mathrm{p}$-value was 0.001 $<\mathrm{p}<0.05$ a post hoc analysis by Bonferroni was performed. Normality testing was done with the Kolmogoroff-Smirnoff test, data were considered as normally distributed if $\mathrm{p}>0.05$. The predictive quality of the different injuries was reported as the area under the receiver operator characteristic curve (AUC). The independent predictive power was analyzed using binary logistic regression. The goodness of fit for the binary logistic regression was analyzed by the HosmerLemeshow test and considered as good if $\mathrm{p}>0.05$. Data were analyzed using IBM SPSS Statistics for Windows software (version 23.0; IBM Corp., Armonk, NY, USA).

\section{Source of bias}

The investigated effect might have many possible cofounders such as ISS, AIS, contamination, preexisting diseases and medication. However, these first data with such a high predictive quality and in this temporary context clearly point into this direction.

\section{Results}

\section{Patient sample and the conditions at admission}

There were significantly more men than women in each ATLS shock class group. The mean age was around forty years, without significant differences (Table 1). The ISS and NISS were significantly increasing along the ATLS shock classifications (Table 1). The physiological parameters, summarized in APACHE II, were rising along the shock severity as well as the according changes in Base Excess, Lactate, Hematocrit, Hemoglobin and Prothrombin Time (Table 1).

\section{The IL 6 secretion pattern in Shock}

The secretion pattern of IL 6 was similar in both distributions, the ATLS shock class and shock index (Figure 1A and B). In both distributions there was a significant increase found according to the initial blood loss. However, the performed post-hoc analysis revealed only significances in ATLS shock class IV vs. I, II and III after 24 hours. No significances were found after 48 hours. Post hoc analysis revealed a significant difference in ATLS shock class I vs. II after 24 hours. A similar statistical situation was found regarding the shock index distribution (Figure 1B). The post hoc analysis revealed significances between shock index $>1.5$ vs. $<0.5$ and $0.5-1.5$ after 24 hours.

\section{The predictive quality of Shock for IL 6 secretion}

The predictive values indicative of exsanguination for IL 6 levels in the multiple injured patients were high. The AUC was increasing according to the shock severity (Figure 2). In patients with a high shock index at trauma site with a maximum of 0.975 (Shockindex: $>1.5$ ) and in the trauma bay with a maximum of 0.931 (ATLS degree: IV) was reached (Figure $2 \mathrm{~B}$ ) after 48 hours.

\section{The interlink between IL 6 secretion SIRS and Sepsis}

The Pearson's correlation showed a clear correlation between Sepsis and class degree of Shock according to ATLS and for the shock index (Table 2). Independent predictive power of IL 6 in regard to SIRS and sepsis demonstrated significant values only for the IL 6 values after 48 hours (Table 3). The ATLS shock classification revealed independent predictive power for Sepsis, however the shock index did not (Table 3).

\section{Outcome Parameters}

Significant differences were found along all groups of the ATLS shock and shock index in this study sample (Table 4). The reduced 
Heining SM (2018) The initial trauma associated hemorrhagic shock in the multiply injured patient has pro-inflammatory effects. A retrospective single center cohort study

Table 1. Characteristics of the patient sample directly at admission. Dimensions are given in square brackets. AIS: Abbreviated Injury Scale [Range1-5, arbitrary]. ISS: Injury Severity Scale [Range 16-75, arbitrary], PT: Prothrombin time, Tc: Platelets. "Kruskal-Wallis H test, ${ }^{\dagger} \chi^{2}$, significant if $\mathrm{p}<0.05$. Kolmogorov-Smirnov $<0.05$ for all groups

\begin{tabular}{|c|c|c|c|c|c|}
\hline ATLS Class & 1 & 2 & 3 & 4 & p-value \\
\hline Sex [male/female] & $265 / 90$ & $100 / 43$ & $24 / 6$ & $12 / 5$ & $<0.001^{\dagger}$ \\
\hline Age [a] & $40.0 \pm 17,8$ & $37,5 \pm 16$ & $40.8 \pm 16$ & $34.4 \pm 12.8$ & $0.389^{*}$ \\
\hline Time to scene $[\mathrm{h}]$ & $6.6 \pm 26.1$ & $3.4 \pm 12.7$ & $1.6 \pm 0.8$ & $2.5 \pm 3.7$ & $0.593^{*}$ \\
\hline AIS Head & $3.1 \pm 1.8$ & $2.7 \pm 2.0$ & $3.3 \pm 1.8$ & $3.4 \pm 1.6$ & $0.235^{*}$ \\
\hline AIS Face & $0.8 \pm 1.2$ & $0.6 \pm 1.0$ & $1.0 \pm 1.6$ & $0.9 \pm 1.3$ & $0.122 *$ \\
\hline AIS Thorax & $1.5 \pm 1.6$ & $2.0 \pm 1.7$ & $2.2 \pm 1.9$ & $3.2 \pm 1.7$ & $<0.001^{*}$ \\
\hline AIS Abdomen & $0.8 \pm 1.5$ & $1.4 \pm 2.0$ & $1.8 \pm 2.1$ & $2.4 \pm 1.8$ & $<0.001 *$ \\
\hline AIS Spine & $0.8 \pm 1.4$ & $1.0 \pm 1.5$ & $1.0 \pm 1.5$ & $1.9 \pm 1.8$ & $0.005^{*}$ \\
\hline AIS Extremities & $1.3 \pm 1.4$ & $1.9 \pm 1.6$ & $2.0 \pm 1.7$ & $1.8 \pm 1.2$ & $<0.001 *$ \\
\hline AIS Pelvis & $0.4 \pm 1.0$ & $0.8 \pm 1.3$ & $0.9 \pm 1.5$ & $1.4 \pm 1.7$ & $<0.001 *$ \\
\hline AIS Skin & $0.4 \pm 0.7$ & $0.5 \pm 0.8$ & $0.6 \pm 0.8$ & $0.8 \pm 0.8$ & $0.239^{*}$ \\
\hline ISS & $26.6 \pm 11.9$ & $32.6 \pm 12.3$ & $38.1 \pm 13.2$ & $40.9 \pm 13.6$ & $<0.001^{*}$ \\
\hline NISS & $36.7 \pm 14.5$ & $42.1 \pm 14.3$ & $48.1 \pm 12.7$ & $50.0 \pm 17.5$ & $<0.001 *$ \\
\hline APACHE II & $13.7 \pm 7.1$ & $16.0 \pm 7.8$ & $19.9 \pm 8.4$ & $19.4 \pm 8.5$ & $<0.001 *$ \\
\hline SAPS II & $30.3 \pm 15.1$ & $38.3 \pm 13.5$ & $27.3 \pm 12.0$ & $31.0 \pm 15.5$ & $0.344^{*}$ \\
\hline Base Excess [mM] & $-2.6 \pm 3.3$ & $-4.6 \pm 4.6$ & $-6.8 \pm 4.7$ & $-5.7 \pm 3.7$ & $<0.001 *$ \\
\hline Lactate $[\mathrm{mM}]$ & $2.5 \pm 1.6$ & $3.2 \pm 2.6$ & $4.2 \pm 3.2$ & $4.2 \pm 2.5$ & $<0.001 *$ \\
\hline Leukocytes $\left[10^{3} / \mu 1\right]$ & $13.4 \pm 5.9$ & $13.0 \pm 5.9$ & $13.3 \pm 6.6$ & $11.6 \pm 6.2$ & $0.791 *$ \\
\hline Hematocrite [\%] & $35.6 \pm 7.0$ & $32.0 \pm 8.5$ & $28.0 \pm 8.6$ & $25.9 \pm 8.7$ & $<0.001 *$ \\
\hline Hemoglobin $[\mathrm{g} / \mathrm{dL}]$ & $12.2 \pm 2.5$ & $10.5 \pm 3.1$ & $9.3 \pm 3.0$ & $8.8 \pm 2.9$ & $<0.001 *$ \\
\hline PT [\%] & $85.0 \pm 15.4$ & $79.0 \pm 18.9$ & $70.0 \pm 20.5$ & $62.0 \pm 29.5$ & $<0.001 *$ \\
\hline $\operatorname{Tc}\left[10^{3} / \mu 1\right]$ & $117.0 \pm 161.0$ & $194.6 \pm 75.7$ & $198.4 \pm 110.5$ & $146.9 \pm 85.0$ & $0.024 *$ \\
\hline
\end{tabular}

Table 2. Pearson's correlation of IL 6 secretion and of the shock degrees on the development of SIRS and sepsis. IL 6 Levels and shock is clearly correlated with septic complications

\begin{tabular}{|c|c|c|}
\hline $\begin{array}{c}\text { Pearson } \\
\boldsymbol{p} \text {-value }\end{array}$ & SIRS & Sepsis \\
\hline IL 6 24h & 0.176 & 0.169 \\
& 0.080 & $\mathbf{0 . 0 0 3}$ \\
\hline IL 6 48h & 0.175 & 0.505 \\
& $\mathbf{0 . 0 0 2}$ & $<\mathbf{0 . 0 0 1}$ \\
\hline \multirow{2}{*}{ ATLS Shock } & 0.074 & 0.153 \\
Shock index & 0.091 & $<\mathbf{0 . 0 0 1}$ \\
\hline
\end{tabular}

Table 3. Binominal Regression analysis of IL 6 secretion on the development of SIRS and sepsis. IL 6 Level after 48 hours and the ATLS shock degree is clearly associated with septic complication in the multiple injured patient. Hosmer Lemeshow test was $>0.05$ for SIRS and sepsis. Data were considered as significant if $\mathrm{p}<0.05$

\begin{tabular}{|c|c|c|}
\hline $\begin{array}{c}\text { p-value } \\
\text { odds-ratio }\end{array}$ & SIRS & Sepsis \\
\hline IL 6 24h & 0.958 & 0.092 \\
& 1.000 & 0.998 \\
\hline \multirow{2}{*}{ IL 6 48h } & 0.073 & $<\mathbf{0 . 0 0 1}$ \\
& 1.009 & 1.004 \\
\hline ATLS Shock & 0.093 & $<\mathbf{0 . 0 0 1}$ \\
& 1.362 & 1.560 \\
\hline Shock index & 0.200 & 0.093 \\
\hline
\end{tabular}

Table 4. The outcome of the patient sample. The dimensions are given in square brackets. *Kruskal-Wallis $\mathrm{H}$ test, ${ }^{\dagger} \chi^{2}$, significant if $\mathrm{p}<0.05$. Kolmogorov-Smirnov $<0.05$ for all groups

\begin{tabular}{|c|c|c|c|c|c|}
\hline ATLS Class & $\mathbf{1}$ & $\mathbf{2}$ & $\mathbf{3}$ & $\mathbf{4}$ & p-value \\
\hline Hosp. [d] & $20.2 \pm 13.1$ & $28.2 \pm 20.3$ & $25.6 \pm 13.3$ & $23.4 \pm 20.0$ & $<0.001^{*}$ \\
\hline ICU stay [d] & $12.3 \pm 10.3$ & $16.0 \pm 14.3$ & $16.3 \pm 10.2$ & $12.9 \pm 11.2$ & $0.010^{*}$ \\
\hline Ventilator [d] & $8.4 \pm 9.3$ & $10.8 \pm 12.7$ & $12.0 \pm 8.7$ & $8.0 \pm 6.4$ & $0.024^{*}$ \\
\hline Mortality [\%] & 18.3 & 19.1 & 19.2 & 41.7 & $0.508^{\dagger}$ \\
\hline
\end{tabular}

Ventilator days in the ATLS Class IV are probably due to the increased mortality in this patient cohort (Table $4 \mathrm{~A}$ ).

\section{Discussion}

SIRS and other multiple trauma-associated complications account for increased hospitalization and worse outcome. In the current study, the question was addressed whether clinical indicators of initial blood loss due to multiple injuries are associated with proinflammatory changes. Previous publications in this field discussed the trauma load, the amount of transfusions, plasma expanders and other resuscitative efforts as indicators for the development of SIRS in the multiple injured patient [5,20-22]. From the view of temporal linearity, all pharmacological resuscitative efforts root in the hemorrhage of multiple trauma. The significant results in these studies might be the logical temporal consequence of hemorrhage [5,20-22]. It is hard to distinguish between the blood loss and trauma load as the reason for the pro-inflammatory state. However, even in patients with a higher ISS the blood loss might be neglectable as a result self-tamponade and low bleeding as depicted earlier $[5,23]$. Blood loss in multiple injured patients significantly increased the pro-inflammatory IL 6 level after 24 and 48 hours and was a good predictor for SIRS and sepsis. The proinflammatory effect of blood loss as the first event in the timeline after trauma seems to be proven. Many cofounders must be assumed in this study and the transfusion regime as a cofounder cannot be excluded definitively. In 48 hours, a plenty of therapeutic procedures take place in a multiple injured patient. Like the definition of SIRS, transfusions are being applied according to clinical guidelines what makes blood loss and transfusion going hand in hand [24,25]. There are several points of view to blood loss and pro-inflammation. The oxygen supply to all vital systems is reduced leading to an endocrine stress reaction resulting in the secretion of epinephrine and administration of norepinephrine by the rescue teams in the hypotonic patient. Maintaining 
A

\section{IL 6 Secretion}

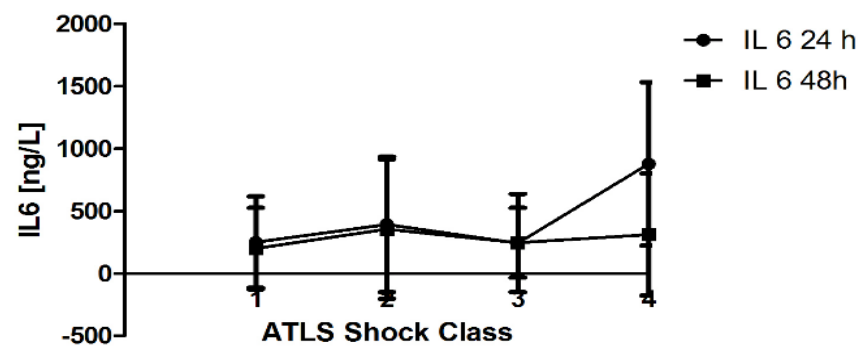

B

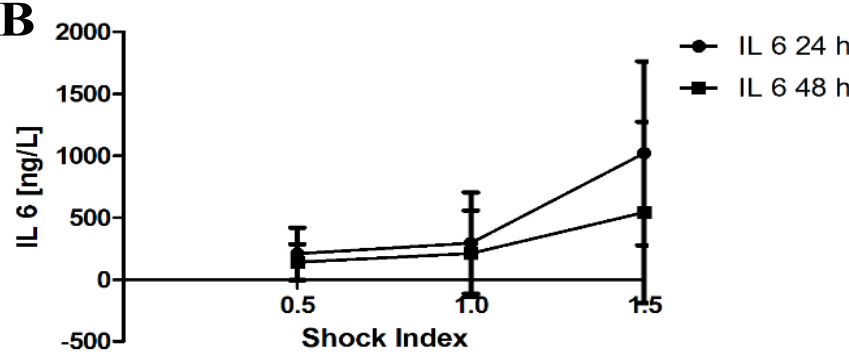

Figure 1. Depicted is the secretion of IL 6 after 24 hours and 48 hours after admission according to the ATLS shock (A) and shock index groups (B). $p<0.001$ for IL 6 between the ATLS groups after 24 hours and $p=0.022$ between the ATLS groups after 48 hours. $\mathrm{p}<0.05$ for IL 6 between the shock index groups after 24 hours and $\mathrm{p}<0.001$ between the shock index groups at trauma site after 48 hours *Kruskal-Wallis $\mathrm{H}$ test. KolmogorovSmirnov $<0.05$ for all groups. (A and B) Bonferroni $\mathrm{p}<0.001$, Schockindex $>1.5$ vs. 0.5 1.5 and $<0.5$; ATLS shock 4 vs. $3-1$

\section{A}

Predictive quality of Shock for IL 6 secretion

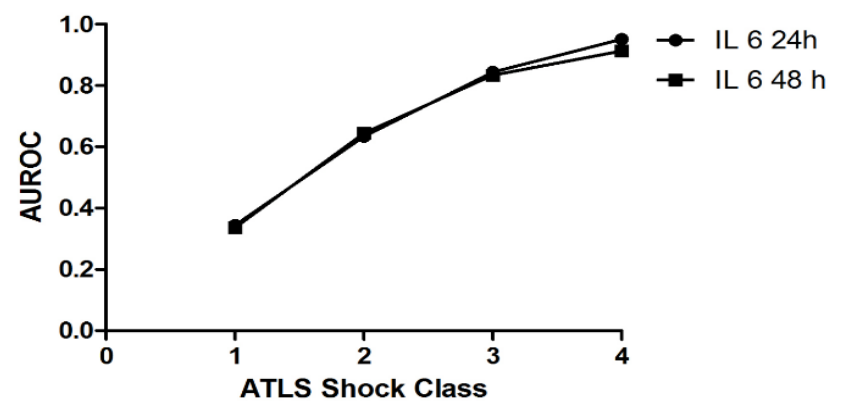

\begin{tabular}{|c|c|c|c|c|}
\hline ATLS Class & $\mathbf{1}$ & $\mathbf{2}$ & $\mathbf{3}$ & $\mathbf{4}$ \\
\hline IL 6 24h AUC & 0.345 & 0.634 & 0.844 & 0.951 \\
\hline IL 6 48h AUC & 0.336 & 0.645 & 0.834 & 0.913 \\
\hline
\end{tabular}

B

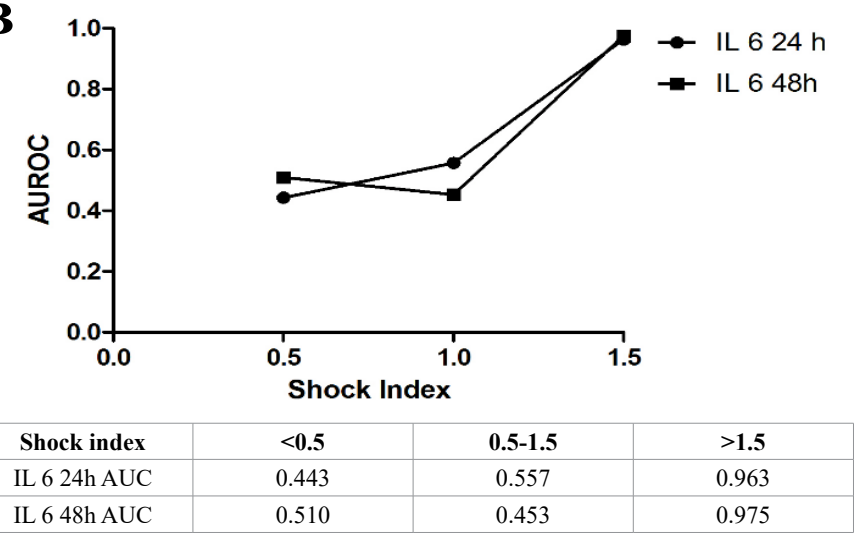

Figure 2. Analysis of the predictive quality of ATLS shock group (A) and shock index group (B) for IL6 secretion if the multiple injured patient. The analysis is given as ROC and given is the area under the curve (AUC), maximal value 1.000 the blood pressure pharmacologically high may result in an additive relative ischemia and stress. Additionally, epinephrine itself increases the systemic IL6 level [26]. Once normalizing the critical physiological state, the multiple injured patient might react with pro-inflammation in the sense of reperfusion. On the other hand, trauma bleeds and exposes hidden antigen that might activate host's primary defense additionally, trauma wounds are polluted leading to an immunological response. Pollution is a complex molecular and corpuscular collection from the outer world. Both, the molecular part by the activation of innate immunity as well as the nano-corpuscular part might activate host's pro-inflammatory response leading to SIRS $[27,28]$. However, in this patient sample no significant difference in the AIS of the skin (Table 1) between the study groups was found putting the blood loss in the center again. As the time sequence from the trauma point on is including all the possibilities of SIRS genesis, the most correct etiology of SIRS, however, might be found experimentally only. The predictive quality of shock in this study is definitively higher than of ISS or NISS as previously published getting closer to the origins of trauma inflammation [5]. The most rational model for the trauma related inflammation could be the overall contribution of all parameters, on the one hand the trauma load and blood loss itself, and on the other hand resuscitative efforts and surgical interventions only regulating the SIRS but never avoiding it. Significant trauma starts with blood loss.

\section{Conclusions}

Class III and IV of Shock and higher levels of shock index states have a higher predictive validity for IL 6 secretion when compared with the shock index at admission in the multiple injured patient. The association between clinical indicators of hemorrhage and IL 6 levels was confirmed, associated with high odds ratio of shock and IL 6 secretion for septic complications. Purposeful clinical consequences lead to quick bleeding control and to damage control transfusion regime.

\section{Funding}

No funding was obtained for this study.

\section{Authorship and contributorship}

All authors contributed equally to this work in the sense of drafting the manuscript, data collection and revising the draft and being part of the whole intellectual work.

\section{Ethics approval and consent to participate}

The study was approved by the Ethics Committee of the University Hospital Zurich with the Permission: "Retrospektive Analysen in der Chirurgischen Intensivmedizin” Nr. St.V. 01-2008.

\section{Conflict of interest}

The authors have no conflicts of interest to declare.

\section{References}

1. Boyle MJ, Smith EC, Archer F (2008) Is mechanism of injury alone a useful predictor of major trauma? Injury 39: 986-992. [Crossref]

2. Søreide K (2009) Epidemiology of major trauma. Br J Surg 96: 697-698. [Crossref]

3. Aneta MB, Grzegorz M, Robert S (2015) Current views on the mechanisms of immune responses to trauma and infection. Cent Eur J Immunol 40: 206-216. [Crossref]

4. Lord JM, Midwinter MJ, Chen YF, Belli A, Brohi K, et al. (2014) The systemic immune response to trauma: an overview of pathophysiology and treatment. Lancet 384: 14551465. [Crossref]

5. Mica L, Furrer E, Keel M, Trentz O (2012) Predictive ability of ISS, NISS, and APACHE II score for SIRS and sepsis in polytrauma patients. Eur J Trauma Emerg Surg 38: 665-671. [Crossref] 

study

6. Ladislav M, Jindrich V, Marius K, Trentz O (2012) The impact of body mass index on the development of systemic inflammatory response syndrome and sepsis in patients with polytrauma. Injury 45: 253-258. [Crossref]

7. Ladislav M, Kaspar R, Marius Keel, et al. (2013) The risk of early mortality of polytrauma patients associated to ISS, NISS, APACHE II values and prothrombin time, a retrospective cohort study. $J$ Trauma Manag Outcom 24:7-6.

8. Sprengel K, Simmen H, Clément MW, Sulser S, Plecko M et al. (2016) Resuscitation with Polymeric Plasma Substitutes is Permissive for Systemic Inflammatory Response Syndrome and Sepsis in Multiple Injured Patients: A Retrospective Cohort Study. Eur $J$ Med Res 21:39. [Crossref]

9. Harrison DA (2012) The Jak/STAT pathway. Cold Spring Harb Perspect Biol 4. [Crossref]

10. Sapan HB, Paturusi I, Jusuf I, Patellongi I, Massi MN, et al. (2016) Pattern of cytokine (IL-6 and IL-10) level as inflammation and anti-inflammation mediator of multiple organ dysfunction syndrome (MODS) in polytrauma. Int J Burns Trauma 1: 37-43. [Crossref]

11. Committee on Trauma, American College of Surgeons Advanced trauma life support for doctors-student course manual 8th ed. Chicago, American College of Surgeons 2008 .

12. Knaus WA, Draper EA, Wagner DP, Zimmerman JE (1985) APACHE II: a severity of disease classification system. Crit Care Med 13: 818-829. [Crossref]

13. Baker SP, O’Neill B, Haddon W Jr, Long WB (1974) The injury severity score: a method for describing patients with multiple injuries and evaluating emergency care. $J$ Trauma 14: 187-196. [Crossref]

14. Champion HR, Copes WS, Sacco WJ, Lawnick MM, Bain LW, et al. (1990) A new characterization of injury severity. J Trauma 30: 539-545. [Crossref]

15. Boyd CR, Tolson MA, Copes WS (1987) Evaluating trauma care: the TRISS method. Trauma Score and the Injury Severity Score. J Trauma 27: 370-378. [Crossref]

16. Le Gall JR, Lemeshow S, Saulnier F (1997) A new Simplified Acute Physiology Score (SAPS II) based on a European/North Amerikan mulficenter study. JAMA 270: 2957 2963. [Crossref]

17. Muckart DJ, Bhagwanjee S (1997) American College of Chest Physicians/Society of Critical Care Medicine Consensus Conference definitions of the systemic inflammatory response syndrome and allied disorders in relation to critically injured patients. Crit Care Med 25:1789-1795. [Crossref]
18. Allgöwer M, Burri C (1967) Shock index. Dtsch Med Wochenschr 92: 1947-1950. [Crossref]

19. Jackson CM, White GC (2001) Scientific and standardization committee communication: a reference system approach to future standardization of laboratory tests for hemostasis.

20. Ferraris VA, Ballert EQ, Mahan A (2013) The relationship between intraoperative blood transfusion and postoperative systemic inflammatory response syndrome. Am J Surg 205: 457-465. [Crossref]

21. Bonde A, Nordestgaard AT, Kirial R, Svenningsen P, Sillesen M (2017) The effect of resuscitation strategy on the longitudinal immuno-inflammatory response to blunt trauma. Injury 48: 2670-2674. [Crossref]

22. Ferraris VA, Brown JR, Despotis GJ, Society of Thoracic Surgeons Blood Conservation Guideline Task Force, Hammon JW (2011) Update to the Society of Thoracic Surgeons and the Society of Cardiovascular Anesthesiologists blood conservation clinical practice guidelines. Ann Thorac Surg 91: 944-982. [Crossref]

23. Gorlinger K, Dirkmann D, Hanke AA, Kamler M, Kottenberg E et al. (2011) Firstline Therapy with Coagulation Factor Concentrates Combined with Point-of-Care Coagulation Testing Is Associated with Decreased Allogeneic Blood Transfusion in Cardiovascular Surgery: A Retrospective, Single-center Cohort Study. Anesthesiology. 115: 1179-1191. [Crossref]

24. Spahn DR, Bouillon B, Cerny V, Coats TJ, Duranteau J, et al. (2013) Management of bleeding following major trauma: an updated European guideline. Crit Care 17: R76. [Crossref]

25. Keller P, Keller C, Robinson LE, Pedersen BK (1985) Epinephrine infusion increase adipose interleukin-6 gene expression and systemic levels in humans. $J$ Appl Physiol 97: 1309-1312. [Crossref]

26. Vidya MK, Kumar VG, Sejian V, Bagath M, Krishnan G, et al. (2018) Toll-like receptors: Significance, ligands, signaling pathways, and functions in mammals. Int Rev Immunol 37: 20-36. [Crossref]

27. Schunck A, Kronz A, Fischer C, Buchhorn GH (2016) Release of zirconia nanoparticle at the metal stem-bone cement interface in implant loosening of total hip replacements. Acta Biomater 31: 412-424. [Crossref]

28. Sahu D, Kannan GM, Vijayaraghavan R (2014) Carbon black particle exhibits size dependent toxicity in human monocytes. Int J Inflam 2014: 827019. [Crossref]

Copyright: (C2018 Heining SM. This is an open-access article distributed under the terms of the Creative Commons Attribution License, which permits unrestricted use, distribution, and reproduction in any medium, provided the original author and source are credited. 